ENCYCLOPEDDIE Encyclopédie berbère

BERBERE

$26 \mid 2004$

26 | Judaïsme - Kabylie

\title{
Kabylie : Anthropologie sociale
}

D. Abrous

\section{(2) OpenEdition}

Journals

Édition électronique

URL : http://journals.openedition.org/encyclopedieberbere/1416

DOI : 10.4000/encyclopedieberbere.1416

ISSN : 2262-7197

\section{Éditeur}

Peeters Publishers

\section{Édition imprimée}

Date de publication : 1 mai 2004

Pagination : 4027-4033

ISBN : 2-7449-0452-X

ISSN : 1015-7344

Référence électronique

D. Abrous, « Kabylie : Anthropologie sociale », Encyclopédie berbère [En ligne], 26 | 2004, document K09, mis en ligne le 01 juin 2011, consulté le 14 décembre 2020. URL : http://journals.openedition.org/ encyclopedieberbere/1416; DOI : https://doi.org/10.4000/encyclopedieberbere.1416

Ce document a été généré automatiquement le 14 décembre 2020.

(c) Tous droits réservés 


\title{
Kabylie : Anthropologie sociale
}

\author{
D. Abrous
}

1 L'organisation sociale kabyle, à l'instar de celle qui caractérise l'ensemble du monde berbère, repose sur deux fondements : un ordre lignager sur lequel se construit un système de fédération. Ce système articule des unités sociales incluses les unes dans les autres: plusieurs lignages (axerrub, adrum) constituent le village (taddart), plusieurs villages se regroupent en tribus (lkerc), ces tribus pouvaient elles-mêmes être confédérées ${ }^{1}$ dans des ensembles plus vastes (taqbilt). Il s'agit là de la description la plus simple qui puisse être donnée de cette organisation sociale. La réalité est cependant plus complexe et plus diversifiée. L'ouvrage de Hanoteau \& Letourneux, qui constitue une référence fondamentale pour la Kabylie du milieu du XIX $x^{e}$ siècle, note que certains villages (exemple: celui de Ighil Imoula de la confédération des Igoujdal) n'étaient rattachés à aucune tribu (Hanoteau 1893, t. 1, p. 4, note $n^{\circ} 1$ ). La tribu elle-même, en dépit de l'ancêtre éponyme auquel elle se rattache, était une fédération politique qui pouvait se démembrer et se restructurer en fonction de l'état des rapports de force (Hanoteau 1893, t. 1, p. 67).

2 La confédération (taqbilt) était le niveau de fédération le plus large mais aussi le plus lâche: les tribus confédérées (car certaines ne l'étaient pas) gardaient entière leur autonomie et le conseil des notables de la confédération, au sein duquel était représentée chacune des tribus confédérées, ne se réunissait qu'en des circonstances exceptionnelles, en particulier en cas de guerre. Ce niveau confédéral fut le premier à être détruit après la conquête française ; à la veille de cette conquête, et probablement depuis le $\mathrm{xvI}^{\mathrm{e}}$ siècle ${ }^{2}$, la Kabylie était traversée par deux grandes ligues (șeff, pl. leșfuf) dénommées «ligue du haut» (șeff $n$ ufella) et la «la ligue du bas» (șeff $n$ wadda). L'ouvrage de Hanoteau (1893, t. 1, p. 19) donne la liste des tribus rattachées à chacune de ces ligues; cette liste montre que les șeff traversaient aussi bien la Haute Kabylie (Igawawen) que la vallée de la Soummam (Tamurt ufella).

Dans les régions de plaine, qui étaient en contact avec des pouvoirs centralisés (dynasties Hafside, beylik de Constantine ou d'Alger), s'étaient constituées des familles qui jouaient le rôle de relais; ce fut le cas de la famille des Aït Kaci (At Qasi) dans la vallée du Sébaou, de celle de Ben Ali Chérif (d'origine maraboutique) dans la vallée de la 
Soummam, et des Aït Mokrane (At Meqqran), famille de «djouad», dans la plaine de la Medjana.

4 La conquête française - car la France fut la première puissance étrangère à avoir totalement conquis la Kabylie et à l'avoir administrée - ébranlera cet édifice social dans ses fondements mêmes. Tous les niveaux ne seront pas atteints de la même manière par ce bouleversement : ce furent les niveaux de fédération les plus larges qui résistèrent le moins bien. La confédération, unité sociale la plus vaste mais la plus lâche, démembrée par les différents découpages administratifs, perdit toute existence politique ; sauf cas exceptionnels (les At Yiraten, par ex.), elle ne constitue, aujourd'hui, même plus un pôle d'identification. Les ligues que l'administration coloniale sut utiliser avec habilité dans la nomination des notables (amin, amin el oumena) finirent par s'essouffler, les nouvelles règles $\mathrm{du}$ jeu politiques rendant leur existence caduque. Ces anciennes ligues ne sont plus, de nos jours, qu'un souvenir lointain dont la mémoire des gens âgés conserve certaines bribes.

Les principales tribus kabyles.

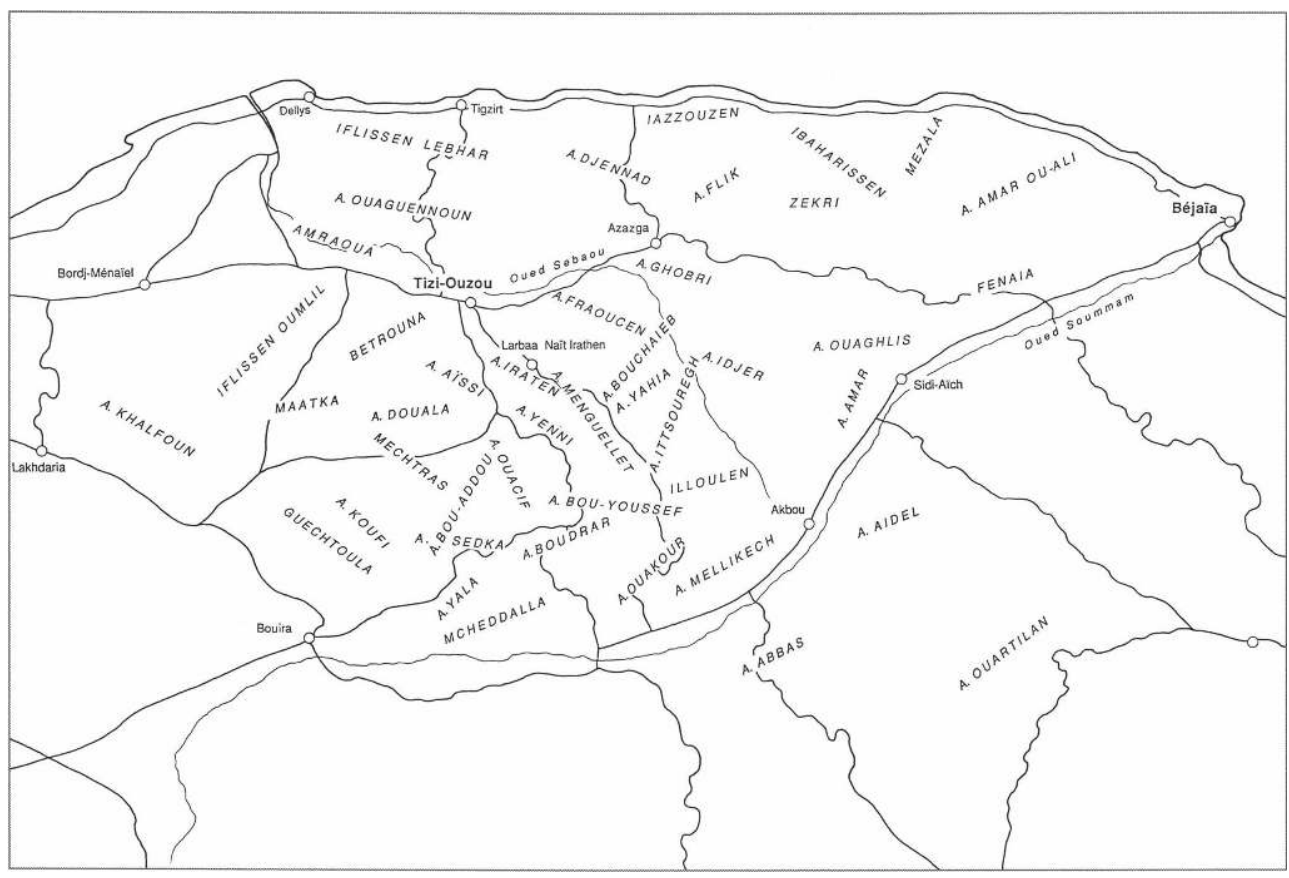

5 Les " grands commandements ", quant à eux - quel que soit le statut social des familles qui les représentaient - furent détruits en deux temps : ceux du Djurdjura furent démantelés immédiatement après 1857 (date de la conquête définitive de la Kabylie) ou dans le sillage du mode d'administration dénommé «la politique kabyle » (Cf. Ageron 1968, t. I, p. 279). Les familles de l'aristocratie guerrière (Aït Mokrane) ou religieuse (Ben Ali Chérif) furent brisées après l'insurrection de 1871; pour la famille Aït Mokrane, ce fut un véritable coup de grâce, son pouvoir politique fut anéanti ; la famille Ben Ali Chérif réussit à survivre mais son pouvoir fut amoindri.

Dans ces profonds bouleversements induits par la conquête française, seules les unités sociales les plus étroites résistèrent au démantèlement. Ces unités, la tribu mais surtout le village, purent résister car c'était en elles que se concentraient les institutions juridiques et politiques sur lesquelles reposait la société kabyle. Le village, soulignent Hanoteau et Letourneux, "est la pierre angulaire de la société kabyle. On y trouve tous les 
éléments qui la composent; c'est là qu'elle se développe et qu'elle vit; c'est là seulement qu'on peut l'étudier dans son ensemble et dans ses détails. Faire connaître le village, c'est donc faire connaître la société entière [...] L'autorité dirigeante du village, la seule, à vrai dire, puisqu'elle possède en même temps que les pouvoirs politique et administratif, la plénitude, au moins en principe, du pouvoir judiciaire est la thadjmaïth ou djemaâ, c'est-à-dire l'assemblée générale des citoyens» (Hanoteau 1893, t. 1, p. 7-8).

7 La tribu présentait aussi des éléments de cohésion (territoire, sanctuaires, marché, solidarité en cas de guerre...) mais à un degré moindre que le village (Hanoteau 1893, t. 1, chapitre XVII). C'est sur ces deux unités, la tribu et le village, que se concentrèrent les efforts de l'administration coloniale. Les principes sur lesquels reposait la gestion administrative de la Kabylie durant la période coloniale ainsi que les différents modes de gestion mis en œuvre depuis 1857 ont été analysés avec précision dans les ouvrages de Ch.-R. Ageron (1968, t. I, notamment chapitre X) et Alain Mahé (2001); seuls seront soulignés ici les points essentiels.

8 L'administration française ne supprima pas les institutions kabyles : la soumission de la Kabylie, négociée entre le Maréchal Randon et les tribus kabyles en juillet $1857^{3}$, garantissait le maintien des institutions ; maintenues donc parce que incontournables, ces institutions seront soumises à un long processus de neutralisation. Trois moments charnière marquèrent ce processus qui fut principalement centré sur la Grande Kabylie.

a) "L'organisation kabyle", devenue effective dès 1858 sous la responsabilité du Maréchal Randon, avait pour objectif de soumettre la djemaa au contrôle de l'autorité française ; elle perdit donc son autonomie politique et financière. Quant à l'amin, bien que le principe de son élection ait été maintenu, il devint un agent au service de l'autorité française; enfin, dans chaque tribu, fut crée un emploi d'amin el-oumena. Ce fonctionnaire était rémunéré, "son rôle doit se borner à renseigner [l] $\left.{ }^{\prime}\right]$ autoritè française, à transmettre ses ordres et à agir suivant ses instructions » (Hanoteau 1893, t. 1, p. 134).

b) L'instauration du « régime civil »

Le maintien des institutions kabyles décidé en 1857 lors de la soumission de la région fut remis en cause par l'insurrection de 1871: bien que placées sous le contrôle de l'autorité française (Cf. supra), les djemaa (aussi bien celles des villages que celles des tribus) avaient pris une part active à l'insurrection. Les amin et surtout les amin el oumena "ont été l'âme de la révolte dans leur pays et se sont fait remarquer par leur ardeur à nous combattre et leur opiniâtreté dans leur résistance ", souligne une lettre envoyée aux autorités militaires de la région (lettre $\mathrm{n}^{\circ} 247 \mathrm{du} 21$ juin 1871) ${ }^{4}$. Après l'écrasement de l'insurrection, le général Chanzy « rattacha l'ouest de la Grande Kabylie, l'arrondissement de Dellys, au territoire civil et créa un vaste cercle militaire de Fort National - Mekla [...] ce cercle fut divisé en 14 sections (18 en 1876) chacune avec sa Djemaa composée de notables nommés (le plus souvent au nombre de douze) et d'un "président " fonctionnaire. Un amin uniquement chargé de renseigner les autorités était proposé à chaque thaddert » (Ageron 1968, t. 1, p. 283). Les prérogatives administratives furent donc supprimées. Les prérogatives judiciaires le furent aussi car après quelques mesures transitoires qui créèrent une situation de flottement (Ageron 1968, t. 1, p. 283), la Grande Kabylie fut rattachée en 1880 au territoire civil et des juges de paix y furent nommés. Ces dernières mesures accentuèrent la mainmise de l'administration française sur les institutions kabyles. Cette « djemaa des douze » (tajmast $n$ țnac) souleva en Kabylie une vive réprobation dont les poètes se sont fait l'écho. Les Kabyles, précise Ageron, « ignorèrent pendant longtemps ces tribunaux. En 1885, nos juges de paix de Kabylie n'avaient encore que dix-neuf affaires à 
instruire dans l'année. Apparemment, comme par le passé, les djemâa ou des arbitres officieux connurent l'essentiel de leurs contestations» (Ageron 1968, t. 1, p. 284-285).

c) Les « centres municipaux »

L'instauration des centres municipaux en 1945 dans les communes mixtes fut la dernière mesure entreprise par les autorités coloniales. L'organisation de ces centres était proche de celles des communes métropolitaines (Morizot 1962, p. 116). Cette réforme toucha principalement la Kabylie: " 150 centres municipaux y furent crées. Seulement 14 douars répartis dans le reste de l'Algérie "en pays arabe" firent l'objet de la réforme " (Mahé 2001, p. 395). L'expérience fut de courte durée car le déclenchement de la guerre d'indépendance en novembre 1954 allait placer la région sous contrôle militaire, mais elle permit aux derniers administrateurs de constater la persistance des institutions kabyles après plus d'un siècle de domination française. Ce fut Jean Morizot qui, affecté en Kabylie en qualité d'administrateur, rendit compte du mode de survie de ces institutions : "Lorsque les Kabyles de la région de Fort-National [Larbâa n At Yiraten] furent invités à se grouper en centres municipaux, ils ont tout simplement reconstitué officiellement les petites républiques berbères d'avant la conquête que nous pensions mortes; leurs limites respectives étaient restés bien vivantes dans la mémoire des gens et les villages et les hameaux se sont regroupés à l'intérieur des centres municipaux presque exactement comme ils étaient autrefois dans la Kabylie indépendante. [...] réserve faite cependant des groupements tribaux et des anciennes confédérations qui ont disparu " (Morizot 1962, p. 117).

Outre cette mémoire relative à l'organisation du territoire, Morizot a décrit avec précision le mode de survie des institutions elles-mêmes. Prenant pour exemple la commune de Fort-National dans laquelle il exerçait les fonctions d'administrateur, Morizot souligne : «Elle présentait une organisation à deux degrés : à l'échelon le plus élevé, il y avait l'organisation officielle, la municipalité [française] qui gérait les intérêts généraux de la circonscription; aux échelons inférieurs, celui des villages, il y avait des organismes occultes, les djemâas (kabyles) qui disposaient de ressources propres, les produits des amendes (occultes); elles avaient leurs agents d'exécution et réglaient avec beaucoup de liberté les affaires locales. Partout les pouvoirs de police, détenus en principe par l'autorité de tutelle, étaient en réalité entre les mains des collectivités villageoises qui veillaient à l'application des qanouns, n'hésitaient pas au besoin à les rénover et jouissaient généralement d'une autorité plus réelle que tout agent assermenté» (Morizot 1962, p. 124).

Jusqu'en 1954, donc, parmi les institutions kabyles traditionnelles, seules ont survécu les djemaas de village, de manière souterraine en parallèle avec le système administratif colonial. En dépit de cette survie discrète, la djemâa jouissait d'une légitimité certaine ; elle n'hésitait pas à rénover les qanouns, ce qui signifie qu'elle ne s'était pas maintenue de manière fossilisée.

$11 \mathrm{Au}$ lendemain de l'indépendance, les villages kabyles, bien que décimés par la guerre, bien que dépeuplés par l'exode rural, ne furent pas désertés et la gestion de la vie quotidienne exigeait un minimum de concertation; sont alors nées ou ont continué à survivre, selon les cas, des djemâas tout à fait informelles en marge des structures officielles, des Assemblés Populaires Communales (Apc), c'est-à-dire des municipalités. Leurs prérogatives ayant été largement érodées, il ne restait plus à ces djemâas que la gestion des travaux d'utilité publique (voirie, adduction d'eau, élec-trification) en raison des lenteurs, voire de l'inefficacité des Apc; dans les villages qui avaient conservé ce rite, la djemaa se chargeait de l'organisation du sacrifice d'automne (timecreț). Ces rares activités que la djemâa continuait à gérer ont permis le maintien 
des anciens liens de solidarité, principalement les travaux collectifs et les cotisations auxquelles participent aussi les émigrés. La djemâa, en survie forcée, était tombée sous la loi du silence à l'instar de tout ce qui relevait de la spécificité berbère.

C'est le mouvement d'avril 1980 et l'ancrage profond de la revendication identitaire en Kabylie qui exercera sur ces "traditions municipales", comme les dénomme A. Mahé, une action de redynamisation. Dans ce processus de redy-namisation, il est possible de repérer trois moments principaux.

a) À partir de 1980, des jeunes (étudiants, enseignants, fonctionnaires), militants pour la plupart du mouvement culturel berbère (МСB) qui venait de naître, "investissent " les villages, commençant timidement des travaux de collecte (lexique, littérature, confections d'herbiers) mais aussi et surtout, assurant les premiers cours informels de berbère. Ces jeunes ne prirent pas directement part à la gestion des affaires des villages mais leur présence et leurs activités évitèrent que les villages se transforment en musée.

b) La relative ouverture consécutive au séisme politique d'octobre 1988 et notamment la nouvelle loi sur les associations ont permis en Kabylie :

- La naissance d'un tissu associatif très dense. Mouloud Kourdache a analysé la manière dont "le mouvement associatif [...] s'approprie les éléments anthropologiques inhérents à la société kabyle et les articule avec le mouvement de reconstruction identitaire» (Kourdache 2001, p. 68). La densité de ce tissu associatif est telle que les villages ont, dans leur quasi-totalité, chacun son association; et dans l'organisation des activités (culturelles le plus souvent), la djemâa est un interlocuteur incontournable.

- La naissance de "comités de villages" avec statut d'association. Ces comités de villages, version rénovée des antiques djemâas, sont animés par des jeunes; ils gèrent une part importante de la vie du village. À la différence des djemâas, leur composition repose non plus sur la structure lignagère mais sur les critères de compétence (au sens moderne du terme) ou sur l'affinité politique.

Sous cet angle-là, la situation des villages kabyles est aujourd'hui assez diversifiée, mais chacun d'eux a au moins une de ces trois structures (djemâa, comité de village, association); le plus souvent, elles coexistent et, dans certains villages, la djemâa traditionnelle n'est plus qu'un groupe de sages (leeqqal $n$ taddart).

On notera aussi parmi ces développements récents la réactivation sporadique de certaines djemâas de tribu : en 1987 celle des At-Jennad et, au début des années 1990, celle des At-Bouaddou ; en 1995 se réunit la djemâa des Illoulen Ousammer ${ }^{5}$, regroupant les trois communes qui la composent: Ighrem, Akbou, Ichellaḍen (Chellata). Ces djemâas de tribu se sont réunies essentiellement pour réglementer les dépenses somptuaires faites à l'occasion des mariages, circoncisions, naissances, retour de pèlerins. Les textes si l'on prend par exemple celui des Illoulen Ousammer (Illulen usammer), sont rédigés en langue française avec les équivalents en kabyle pour les motsclé; les cérémonies et les dépenses sont réglementées dans le moindre détail, des sanctions sont prévues. Dans le cas des Illoulen Ousammer, cette dynamique enclenchée en 1995 a donné naissance en février 2001 à une structure permanente : «La coordination des notables des villages de la commune d'Ighrem ». Il s'agit là de simples indications, ces tentatives récentes de regroupement au niveau supra-villageois nécessiteraient des monographies précises.

c/ Le soulèvement d'avril 2001

En dehors des moments de crise, ces institutions villageoises, qui constituent un maillage serré en Kabylie, servaient de support à la revendication identitaire 
(financement d'activités culturelles, organisation de festivals poétiques, de cours de langue...); en période de crise, elles servent de cadre de mobilisation. Le soulèvement qui a secoué la Kabylie à partir d'avril 2001 en donne une parfaite illustration : se substituant aux partis politiques (notamment le FFs et le RCD fortement implantés dans la région), les djemâas et comités de villages ont organisé les marches, réquisitionné les moyens de transport, coordonné la solidarité avec les blessés (prise en charge, achat de médicaments...) et avec les familles des victimes, assuré une aide financière régulière (comme c'est le cas des villages de l'Akfadou) aux familles des détenus.

Le mouvement né de ce soulèvement porte le nom de Coordination des Archs, Daïras et Communes; les médias et le pouvoir le désignent par "mouvements des Archs" (« arouch» au pluriel). "Arch» désigne en kabyle la tribu. Ce mouvement a, en effet, réactivé certaines assemblées de tribus comme celles des At Jennad, At Yiraten, At Ghobri.

14 L'analyse de ce mouvement horizontal né en plein soulèvement reste à faire, on peut seulement souligner ici que sa très forte capacité de mobilisation au moment où la crise avait atteint son paroxysme (organisation de la marche du 14 juin 2001, blocage totale des élections du 30 mai 2002) vient de son lien très étroit avec les djemâas et surtout les comités de villages. Ce soulèvement dont toutes les conséquences ne peuvent pas encore être analysées constituera très probablement un tournant dans l'histoire de la Kabylie.

Le lien entre la revendication identitaire et le socle anthropologique ne se limite pas aux institutions; cette revendication puise aussi dans le système de valeurs, en particulier dans le sens de l'honneur : la lutte pour la culture berbère est perçue comme un défi à relever, une question d'honneur. Durant le boycott scolaire et universitaire en 1994-1995, un mot d'ordre largement affiché en Kabylie et particulièrement mobilisateur affirmait «tamaziyt $d$ nnif: tamazight est une question d'honneur, i.e. de dignité » (Abrous 1995, p. 584).

16 Les travaux d'Alain Mahé montrent avec précision que « la sacralité de la harma du village a été peu affecté par les évolutions multidimensionnelles qu'a connues la tajmaat » (Mahé 2001, p. 490). C'est le caractère sacré de l'espace villageois qui explique, par exemple, la réactivation de l'institution des vigiles ${ }^{6}$ en temps de crise (en particulier : avril - juin 2001 et mars 2002, mai 2002).

17 Enfin, ce processus de reconstruction identitaire en Kabylie puise abondamment dans la mémoire de la région. Le chapitre consacré par Mouloud Kourdache à la dénomination des associations est, à cet égard, très significatif : la référence va du saint local (Jeddi Abdelmalek) au militant berbèro-nationaliste (Imache, Laïmèche) en passant par les poètes (Si Mohand ou Mohand, Youcef ou Qasi), les écrivains (Mouloud Feraoun, Taos Amrouche) et les bandits d'honneur (Abdoun, Ahmed ou Merri); le nom d'Arezki Lbachir, illustre bandit d'honneur, est perpétué par deux associations : l'une dans son village natal à Aït Bouhini, l'autre à Azazga, ville dans laquelle il fut guillotiné avec cinq de ses compagnons. La référence à ces noms est d'autant plus significative qu'aucun d'entre eux, à l'exception de celui de Mouloud Feraoun, n'est transmis par l'école. Il s'agit bien, comme le soulignait Salem Chaker de la « construction d'une identité en rupture " (Chaker 1985).

18 Ce socle anthropologique en Kabylie est aujourd'hui, au même titre que la langue et la littérature, traversé par une puissante dynamique de renouvellement. La société aussi 
bien que la langue négocient leur rapport au changement, et donc leur survie, dans une multiplicité de formes qui prouve toute la complexité de cette négociation.

\section{BIBLIOGRAPHIE}

ABRous D., 1988, « Leenaya » Encyclopédie berbère V(p. 633-635), Édisud, Aix-en-Provence.

ABRous D., 1995, « Djemâa - tajmazt » Encyclopédie berbère XVI (p. 2434 - 2438), Édisud, Aix-enProvence.

ABRous D., 1995, « Le Haut Commissariat à l'Amazighité ou les méandres d'une phagocytose », Annuaire de l'Afrique du Nord, XXXIV, Édition du CNRS, Paris.

AGERON Ch.-R., 1968, Les Algériens musulmans et la France (t. I), (1871-1919), Paris, P.U.F. (notamment $\mathrm{p}: 267-292)$.

CARREY E., 1994 (réédition), Récits de Kabylie. La conquête de 1857, Épigraphe, Alger.

CHAKER S., 1985, « La construction d'une identité en rupture : Langue, écriture et culture dans le domaine berbère », Université de Montpellier, Symposium international : La production d'identité, $p$ : 41-56.

DAUMAS M., 1847, La Grande Kabylie : études historiques, Hachette et $\mathrm{C}^{\mathrm{ie}}$, Paris.

DEVAUX C., 1859, Les Kebailes du Djurdjura. Études nouvelles sur le pays vulgairement appelé Grande Kabylie, Challamel, Paris.

GENEVoIS H., 1995, Monographies villageoises I : At Yanni et Taguemount-Azouz, Édisud / La boîte à documents, Aix-en-Provence.

hanoteau A. \& Letourneux L., 1893, La Kabylie et les coutumes kabyles - t. I, Challamel, Paris.

(Réédition : 2003, Paris, Éditions Bouchène.)

IBN KHALDoun A., 1925, Histoire des Berbères et des dynasties musulmanes de l'Afrique septentrionale (traduit de l'arabe par De Slane) - t. I, Geuthner, Paris.

KOURDACHE M., 2001, Mouvement associatif et reconstruction identitaire en Kabylie, Université de Béjaïa, Béjaïa, Mémoire de Magister sous la direction de Salem Chaker et Dahbia Abrous.

MAHE A., 1994, Anthropologie historique de la Grande-Kabylie XIX ${ }^{e}$ et XX ${ }^{e}$ siècle : histoire du lien sociale associatif, Paris, École des Hautes Études en Sciences Sociales (Ehess), Thèse de Doctorat sous la direction de Cornélius Castoriadis ; publiée sous le titre Histoire de la Kabylie, $\mathrm{XIX}^{e}-\mathrm{XX}^{e}$ siècles, Paris, Éditions Bouchène, 2001.

MAMMERI M., 1980, Poèmes kabyles anciens, Maspéro, Paris.

MASQUERAY E., 1983 (réédition), La formation des cités chez les populations sédentaires de l'Algérie : Kabyles du Djurdjura, Chaouïa de l'Aurès, Béni-Mzab, Édisud, Aix-en-Provence.

MORIZOT J., 1962, L'Algérie kabylisée, éd. Peyronnet et Cie, Paris. 
RoBIN J.-N., 1999, La Kabylie sous le régime turc, (présentation et notes de Alain Mahé), Bouchène, Paris.

\section{NOTES}

1. Ibn Khaldoun (Histoire des Berbères, t. I) évoque les Zouaoua en page 173, 185, 255, 256, 292, 293 , 298, 298, 299 ; il énumère, en page 256, les noms des tribus et confédérations zouaoua « les plus marquantes » à son époque.

2. Hanoteau et Letourneux (t. I : 17-18) font remonter l'origine de ces ligues à des dissensions qui avaient divisé la famille des « Aït El Kadhi » (At Lqaḍi).

3. Emile Carrey (1994: 66 et 67) rapporte en ces termes les propos du maréchal Randon lors de la soumission des At-Yiraten et des tribus qui les avaient aidés à défendre les dernières positions : «Vous pourrez, comme par le passé, vous choisir des amins mais ils doivent être reconnus et investis par la France; vous pourrez même garder vos institutions politiques de villages pourvu que vos chefs sachent vous maintenir en paix». Ces propos concentrent les principes du système dénommé «organisation kabyle ".

4. Voir Les Archives d'Outre-Mer, Aix-en-Provence : Fonds des bureaux arabes, cercle de Tizi-Ouzou, registre de correspondance $n^{\circ} 31 \mathrm{II} / 17 \mathrm{du} 2$ janvier au 18 octobre 1871; dans ce registre, les lettres $n^{\circ} 247 \mathrm{du}$ 21 juin 1871, $n^{\circ} 273$ du 5 juillet 1871, $n^{\circ} 286$ du 9 juillet 1871, $n^{\circ} 290$ du 10 juillet 1871 etc. contiennent des détails très précis sur les mesures de répression : débats sur le mode d'application du séquestre, listes d'otages retenus jusqu'au paiement de la contribution de guerre par les villages et/ou tribus, et surtout de longues listes de prisonniers « dangereux à éloigner du pays » (Lettre $n^{\circ} 290 \mathrm{du} 10$ juillet 1871) parmi lesquels de nombreux amin et amin el oumena. Ce fonds contient aussi quelques lettres détaillées portant sur la nécessité de revoir le fonctionnement des djemaa; lettre $n^{\circ} 372$ du 10 août 1871 et lettre $n^{\circ} 376$ du 11 août 1871.

5. Pour les données relatives aux Illoulen Ousammer, voir: H. Bouzida et F. Akhenak, octobre 2003 : Taynarrat di tmurt $n$ Leqbayel: taddart $n$ Tzayart; tama $n$ Bgayet, tajiwant $n$ yeyrem (Aqbu). Mémoire de fin de licence, sous la direction de A. Ould Fella, Département de langue et de culture amazigh, Université de Béjaïa. (Monographie du village de Tazaghart).

6. Sur l'institution des vigiles, voir Henri Genevoix 1995: Monographies villageoises (At Yanni et Taguemount - Azzouz), (page 115), Édisud, Aix-en-Provence.

\section{INDEX}

Mots-clés : Colonisation, Ligues, Kabylie, Organisation sociale, Répression, Tribu 